\title{
ADVANTAGE OF CORE NEEDLE BIOPSY OVER FINE NEEDLE ASPIRATION CYTOLOGY IN PALPABLE BREAST LUMPS
}

\author{
Guddi Rani Singh ${ }^{1}$, Manoj Kumar Rai², Reecha Singh ${ }^{3}$, Bipin Kumar', Vijayanand Choudhary5, Ajit Kumar6, Suryajit Kumar Singh ${ }^{7}$
}

${ }^{1}$ Senior Resident, Department of Pathology, Indira Gandhi Institute of Medical Sciences, Patna.

2 Professor, Department of Pathology, Indira Gandhi Institute of Medical Sciences, Patna.

${ }^{3}$ Associate Professor, Department of Pathology, Indira Gandhi Institute of Medical Sciences, Patna.

${ }^{4}$ Professor, Department of Pathology, Indira Gandhi Institute of Medical Sciences, Patna.

${ }^{5}$ Additional Professor, Department of Pathology, Indira Gandhi Institute of Medical Sciences, Patna.

${ }_{6}^{6}$ Senior Resident, Department of Pathology, Indira Gandhi Institute of Medical Sciences, Patna.

${ }^{7}$ Senior Resident, Department of Pathology, Indira Gandhi Institute of Medical Sciences, Patna.

\section{ABSTRACT}

\section{OBJECTIVE}

To study the advantage of Core Needle Biopsy (CNB) over Fine Needle Aspiration Cytology (FNAC) in palpable breast lumps by comparing the diagnostic accuracy in terms of sensitivity, specificity, positive and negative predictive values considering the histopathological diagnosis of incised or excised specimen as the gold standard.

\section{METHODS}

A prospective study of 100 female patients with palpable breast lump was conducted in the Department of Pathology of a tertiary care teaching hospital of Northern part of India. All the patients were randomly selected, irrespective of their age, religion, marital status, occupation or social status for FNAC following a thorough clinical check-up. During the study period, 54 patients were selected for core needle biopsy on the basis of insufficient FNAC material for making definitive diagnosis and for sub-typing the tumours. All core biopsy samples were subjected for histopathological examination. The diagnostic findings were compared with the histopathological findings of the lumpectomy or mastectomy specimen and with those of FNAC findings for each and every patient.

\section{RESULTS}

The sensitivity of FNAC and CNB was $96.29 \%$ and the specificity for malignant lesions was $100 \%$. The positive predictive value was $100 \%$ and the negative predictive value was $95.12 \%$.

\section{CONCLUSION}

There is no significant difference in the diagnostic accuracy between core needle biopsy and FNAC.

\section{KEYWORDS}

Core Needle Biopsy, Fine Needle Aspiration Cytology, Palpable Breast Lump.

HOW TO CITE THIS ARTICLE: Singh GR, Rai MK, Singh R, et al. Advantage of core needle biopsy over fine needle aspiration cytology in palpable breast lumps. J. Evolution Med. Dent. Sci. 2016;5(67):4759-4763, DOI: 10.14260/jemds/2016/1085

\begin{abstract}
INTRODUCTION
With growing awareness in the general population, especially about breast pathologies, a significant number of female patients presented with breast lump in outpatient departments. Clinical examination is followed by diagnostic procedures, which include the invasive investigative procedures such as Fine Needle Aspiration Cytology (FNAC), Core Needle Biopsy (CNB) and histopathological examination of incisional or excisional biopsy specimen.(1)

In case of palpable breast nodules, FNAC is a well-established method for the diagnosis of breast lesions. It has the advantages of being highly accurate in experienced hands, cost effective and useful for small lesions not eligible for CNB.(2) Its limitations are represented by the lack of experienced cytologists in many institutions, the inability to reliably distinguish invasive from in situ carcinoma and the
\end{abstract}

Financial or Other, Competing Interest: None.

Submission 21-04-2016, Peer Review 29-07-2016,

Acceptance 05-08-2016, Published 19-08-2016.

Corresponding Author:

Dr. Reecha Singh,

E2/2 Quarter No. Indira Gandhi Institute of Medical Sciences,

Patna-14.

E-mail: sreecha@ymail.com

DOI: 10.14260/jemds/2016/1085 features in breast aspirates with the histological classification system used as the "gold-standard," particularly in benign lesions.(3)

In the recent years, the use of CNB for the non-operative diagnosis of breast carcinoma has been generally favoured over the alternative of Fine Needle Aspiration (FNA). This is based on the fact that core biopsy allows evaluation of both cytological and architectural features and provides a definitive diagnosis of invasive carcinoma on one hand and a benign on the other. It also allows for easier identification of micro-calcifications. Furthermore, it reduces the number of inadequate samples and requires a lesser degree of diagnostic expertise.(4) Core needle biopsy is however not without disadvantages. These include a high cost (When compared with FNAC), long tissue processing time, patient discomfort such as pain and haematoma, and the risk of seeding of the tumour along the needle track. $(5,6)$

CNB has been reported to achieve better sensitivity and specificity, especially in non-palpable lesions that appear as not definitively benign or malignant. The average sensitivity of FNAC in palpable breast lump is approximately $87 \%$, the specificity close to $100 \%$, the predictive value of a positive diagnosis nearly $100 \%$ and the predictive value of a negative 
diagnosis between $60 \%$ and $90 \%$. Diagnostic accuracy of CNB is between 97 and $100 \% .(7,8)$

Hence, this study was undertaken to see the advantage of CNB over FNAC for the palpable breast lump and to highlight the drawbacks of blind core biopsy over assisted core biopsy.

\section{AIMS AND OBJECTIVES}

To compare the diagnostic accuracy of CNB over FNAC in term of sensitivity and positive predictive value for the entire study and specificity and negative predictive values in relation to the malignant lesions considering the histopathological diagnosis of incisional or excisional biopsy specimen as the gold standard.

\section{MATERIALS AND METHOD}

This prospective study was conducted in the Department of Pathology of Tertiary Care Hospital of Northern part of India between the period of August 2011 and October 2012. Fifty four female patients attending the surgical outpatient department of our hospital were selected keeping in mind the criteria mentioned below.

\section{Inclusion Criteria}

- Age between 10 and 70 years.

- Palpable breast lump of variable duration.

\section{Exclusion Criteria}

- Patient not willing (Written informed consent taken).

- Frank malignant mass with skin infiltration.

FNA of 54 selected cases with palpable breast lump were performed free handed using a 23-G needle attached with 10 $\mathrm{mL}$ disposable plastic syringe. The sample material was smeared and stained with May-Grunwald-Giemsa and Papanicolaou stain.

In case of CNB an anaesthetic drug (Xylocaine) was locally injected before the procedure, then a $14-G$ needle was used for the sampling and 5 to 7 fragments were sampled in all cases and the specimen immediately fixed in formalin solution.

Results of both FNAC and CNB were compared with the diagnostic histopathological findings of incisional or excisional biopsy specimen as gold standard and sensitivity, specificity, Positive Predictive (PPV) and Negative Predictive Value (NPV) values were calculated.

\section{OBSERVATION AND RESULTS}

The observations and results of our study were tabulated and analysed as below:

\begin{tabular}{|c|c|c|}
\hline $\begin{array}{c}\text { Age Groups } \\
\text { (In Years) }\end{array}$ & $\begin{array}{c}\text { No. of } \\
\text { Cases }\end{array}$ & $\begin{array}{c}\text { Percentage of } \\
\text { Cases }\end{array}$ \\
\hline Less than 20 & 6 & 11.11 \\
\hline $21-40$ & 22 & 40.74 \\
\hline $41-60$ & 23 & 42.59 \\
\hline $61-80$ & 2 & 3.70 \\
\hline$>80$ & 1 & 1.85 \\
\hline \multicolumn{2}{|c|}{ Table 1: Distribution of Cases in } \\
Different Age Groups (54 Cases) \\
\hline
\end{tabular}

In this study, the maximum number of 23 cases (42.59\%) was found to be within age group of 41-60 years followed by 22 cases $(40.74 \%)$ in the age group of $21-40$ years; 6 cases
(11.11\%) were from less than 20 years' age group, while only 3 cases $(5.55 \%)$ were from above 60 years' age group.

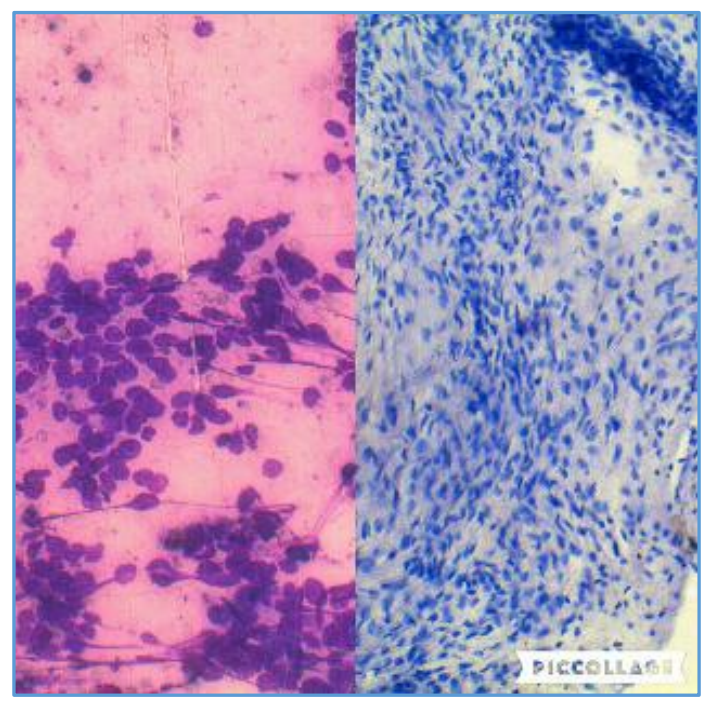

Fig. 1: FNA \& CNB - Showing Benign Phylloid Tumour

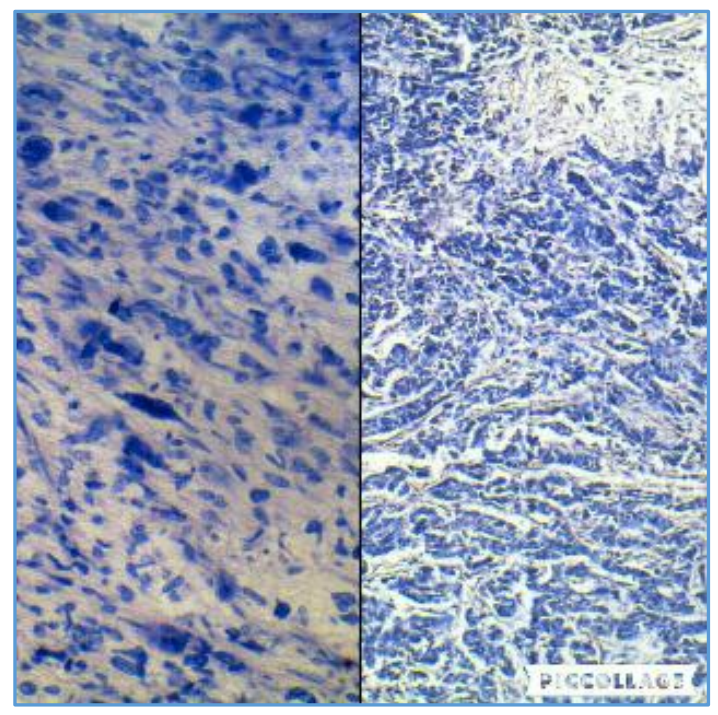

Fig. 2: FNA \& CNB - Showing Malignant Phylloid Tumour

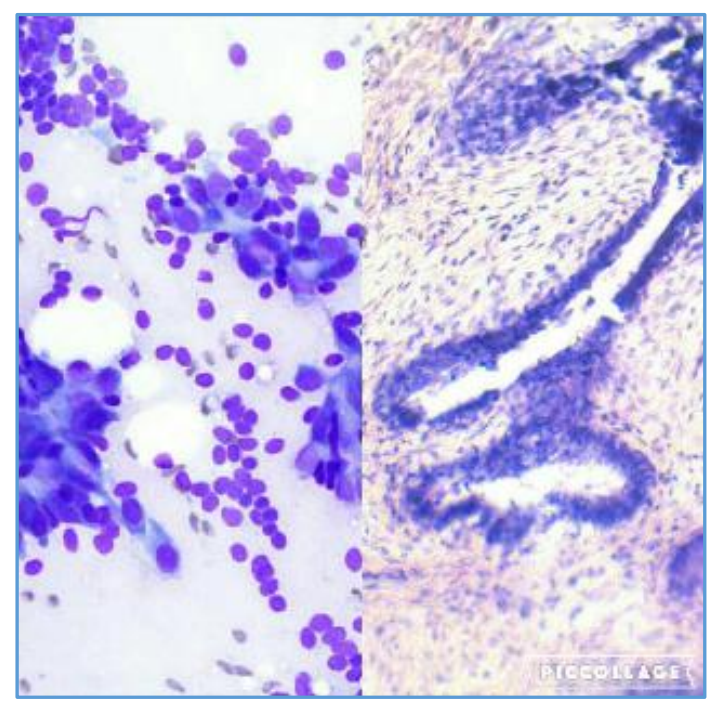

Fig. 3: FNA and CNB - Showing

Fibroadenoma of Breast 


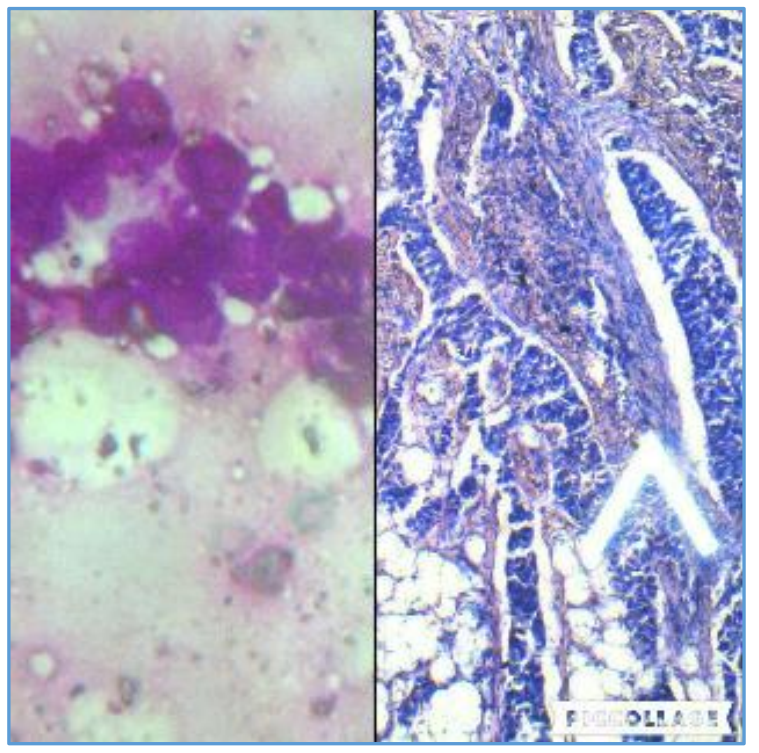

Fig. 4: FNA \& CNB - Showing Fibroadenoma of Breast

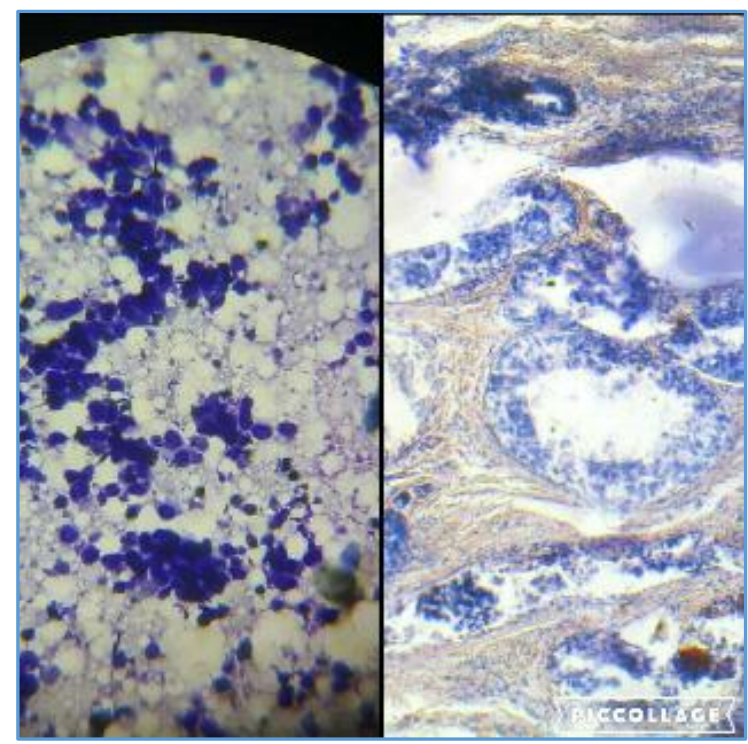

Fig. 5: FNA \& CNB - Showing Ductal Carcinoma in Situ

\section{DISCUSSION}

A lump in the breast with anxiety regarding a possible malignancy is a common complaint, presenting in the surgical outpatient department of all major hospitals. (9) Hence, a quick diagnosis of a lump in the breast is essential.(9) Criteria such as cost effectiveness, use of anaesthesia, time between the diagnostic procedure and report, patient's hospital stay and most importantly reliability in deciding subsequent treatment are all factors to be taken into account in this regard.(9) Considering patient's comfort, lack of requirement of anaesthesia, rapid analysis and reporting and an absence of false positive results makes FNAC an ideal initial diagnostic modality in breast lumps.(9) The expansion of FNAC in the primary diagnosis of cancer in the last 30 years has been enormous and hugely successful. Its use in detecting the presence of cancer before surgery and as a guide to rational treatment has been well documented.( 9 )

The present study was done on 54 cases of breast lump to determine the advantage of core biopsy over fine needle aspiration biopsy. All patients were firstly subjected to FNAC followed by core needle biopsy. Both reports were then matched with final incisional or excisional biopsy report to assess their sensitivity, specificity and predictive value. The results obtained were tabulated and conclusions drawn based on statistical tests. Though many aspects relating to the patient profile were tabulated and compared with those in other similar studies, the most important aspect of this study was to draw conclusions regarding the cytohistological correlation in these patients.

Age of the patients in this study varied from less than 20 years to 80 years and above. The youngest patient with palpable breast lump was 16 years. The highest incidence of breast lump was observed in the age group of 41-60 years. In the present study, maximum number of carcinoma breast was recorded in the group of 41-60 years, although benign lesions were seen in patients of all age groups, but were more commonly seen in younger age groups. In a similar study done by Hussain(10) on 50 patients, the age distribution was between fifteen and sixty-five years and the maximum patients were seen in the thirty one to forty years' group (30\%). Similar studies done by Homesh et al,(11) Arwiga et al,(12) and Tiwari et al(13) showed similar age patterns.

In the present study, it was observed that 33 cases $(61.11 \%)$ of breast lump was found on right side and more were malignant lesions; 20 cases were from left side and more were benign lesions.

In the present study, 42 cases out of 54 cases were multiparous and 10 cases (18.51\%) have positive family history. This corresponds to $74 \%$ of patients in comparison to $69.3 \%$ of 296 patients who were married in the series by Homesh et al(11) Thus, in both series more married women presented to the outpatient department than unmarried ones. Though not directly related to our study, these figures may indicate the relative reluctance of young unmarried women to present to the outpatients department for a breast examination, since benign breast lumps, especially variants of Aberrations of Normal Development and Involution (ANDI) are definitely not uncommon in the young unmarried patient. Also only 7 of our married patients were nulliparous and all those who had children had breastfed their babies.

FNAC and CNB represent the most widely used methods for pathological diagnosis of breast nodules, both with their specific advantages and limitations. The overall sensitivity and specificity of FNAC and CNB in the classification of breast lesions depend on the radiological and histological features and on specific variables intrinsic to the technique. In most cases, CNB has both higher sensitivity and specificity than FNAC in diagnosing benign and malignant lesions.(14-16) However, as reported by Willems et al, the studies which reported high sensitivity (97.1\%), specificity (99.1\%), PPV (99.3\%) and NPV (96.2\%) included only definitive benign and malignant lesions and excluded the atypical and suspicious categories.(15,16) In fact, Westenend et al reported that the PPV of FNAC for malignancy was comparable with $\mathrm{CNB}$, but decreased for suspicious lesions and in case of atypia.(17)

Besides, CNB allows the discrimination between in situ and invasive lesions and is a more accurate method to distinguish between invasive lobular and invasive ductal carcinoma based on histological and immuno-histochemical features. This preoperative distinction can be relevant for planning the extent of the surgical approach for the choice of an adequate chemotherapy and for the increased risk of 
contralateral disease in the case of invasive lobular carcinoma. $(16,18)$

As regards to technical aspects, FNAC is more suitable for lesions close to the chest wall, vessels and implant, for very small or deep and difficult to reach lesions and for patients on anticoagulants. As a general feature of cytology, good quality FNAC depends on the competence of the aspirator and its interpretation is primarily determined by the experience of the pathologist. $(16,19,20)$

Besides, the success rate of FNAC for obtaining a definite diagnosis also depends both on the palpability and size of the lesion. FNAC has average success rates of $75-90 \%$ for palpable and $34-58 \%$ for non-palpable breast lesions, whereas success rates reported for CNB are $97 \%$ and $94 \%$, respectively.(12,16,21) Another important criterion is represented by the lesion size. FNAC has a success rate of only $50 \%$ for lesions less than $10 \mathrm{~mm}$, while CNB is successful in over $90 \%$ of such lesions. Therefore, the success rate of FNAC seems to be especially low for non-palpable lesions and for those smaller than $10 \mathrm{~mm}$. Moreover, FNAC accuracy rates are also decreased for large tumours and for calcified lesions, because of an higher rate of insufficient sampling than masses. $(16,22)$

The main advantages of FNAC are minimal invasiveness, reduced cost, pathological assessment of small lesions which are not amenable to CNB. Moreover, it allows same day diagnosis of breast cancer and the identification and management on the same day of those patients with benign disease.(1)

Therefore, FNAC should be considered as the first method to evaluate breast lesions, recognized by means of imaging techniques; CNB should be performed for unanswered diagnostic cases (C1-C3) and when it is necessary to have such information as invasiveness or histological type of breast lesion.

Besides, Capalbo et al recently reported that the presence of the pathologist on site could allow to obtain high rate of adequate samples and to reach a diagnostic concordance between FNAC and histology of $98.1 \%$.(12)

In our experience, comparable results for FNAC and CNB were obtained in terms of sensitivity $(96 \%$ vs $96 \%)$, specificity ( $100 \%$ vs $100 \%)$, diagnostic accuracy (100\% vs $100 \%$ ) and NPV (94.2\% vs $94.11 \%$ ). As for any diagnostic procedure, a higher NPV is important to minimize undertreatment and it was achieved by CNB biopsy.

Therefore, based on our study, results of both FNAC and CNB were almost same.

On the contrary despite advances in biopsy devices and techniques, false-negative diagnoses still remain unavoidable and may delay the diagnosis and treatment of breast cancer. The most common reasons for false-negative diagnosis are represented by technical or sampling errors, failure to recognize or act on radiologic-histological discordance, and the lack of imaging follow-up after a benign biopsy result. Technical difficulties (poor lesion or needle visualisation, especially after the injection of local anaesthetic drug, deeply located lesions, dense fibrotic tissue) cause inaccurate sampling, but can be reduced by using modified standard techniques.(22)

Optimisation of technique, radiologic-histological correlation and post-biopsy follow-up protocols are recommended in order to reduce the occurrence of falsenegative diagnosis at US-guided CNB performed by radiologists. $(10,11,23)$ In our experience, the number of false negatives resulted to be similar for both FNAC and CNB (2 vs 3 , respectively) and the definitive histological control was mandatory in case of highly suspicious radiological features (Invasive ductal carcinomas in all cases).

Our study has some important limitations, mainly represented by the small number of enrolled patients, the potential bias for patient recruitment basing on the selection criteria of the study, the impossibility of evaluating the reproducibility of each technique and the inter-observer variability, the lack of a direct confrontation between FNAC and CNB for each lesion, the minimal inhomogeneous sample size for FNAC and CNB and the lack of a confrontation with mammographic lesions. (24)

Expertise of the person performing and interpreting the fine-needle aspiration often influences results. Yeoh et al(23) from Hong Kong reported a high proportion of unsatisfactory samples (48\%) with doctors who performed FNAC occasionally. Patel et al(25) showed that FNAC results were influenced by the number of needle manoeuvres performed with less than CNB occasionally offered additional information. The slight advantage was due to availability of tissue from the first and often the only pass for assessment and performance of ancillary tests, which obviated the need for further sampling. The conditions which have a risk of a false positive result are papillary lesions, atypical epithelial hyperplasia, regenerating epithelial atypia and atypia of ductal epithelium in a cyst.

A risk of false negative results exists in low-grade malignancies, small or complex proliferative lesions as well as in tumours with central necrosis or a small cell carcinoma. Silverman et al(26) concluded that for palpable lesions, FNAC as compared to core biopsy appears to have more predictive value in confirming the diagnosis of carcinoma and locally recurrent disease.(27)

\section{Classification Wise Incidence of Breast Carcinoma}

T. Philip et al (1996) reported $60 \%$ cases of invasive ductal carcinoma (NOS).(28)

Mohan H (2005) and Rosai J (2004) reported 70\%, 80\% and $75 \%$ cases of invasive ductal carcinoma (NOS) respectively. $(29,30)$

The above data shows the incidence of histopathological classification-wise distribution of breast carcinoma studied by different workers of different parts of the world, which reveals that invasive ductal carcinoma (NOS), is more common breast cancer than any other types of breast cancer. In the present study, similar finding of breast carcinoma incidence was noticed, i.e. invasive ductal carcinoma (NOS) being the most common variety $(69.56 \%) ; 13.04 \%$ were invasive lobular carcinoma, $4.34 \%$ were medullary carcinoma of breast and $8.69 \%$ were ductal carcinoma in situ. Other types were not found probably due to small number of cases and lack of followup.

\section{CONCLUSION}

FNAC and CNB represent accurate methods for the characterisation of palpable breast nodules with similar values of diagnostic accuracy, sensitivity, specificity and NPV. 
In experienced hands, FNAC could be still considered the first method to evaluate breast lesions being less invasive. CNB has a higher PPV and should be performed for uncertain diagnostic cases and when the evaluation of the invasiveness or histological type of breast lesion is mandatory.

Both are sampling methods and have their advantages and limitations and their specific indications. But CNB has its own limitations also that restricted its use.

It requires operation theatre. It can only be done under local anaesthesia. It is a time taking process from fixation, processing and staining of slide. Core biopsy failure may occur due to inadequate sampling, needle entry into normal site, cell morphological alteration by compression by the hollow canal.

To overcome these failures, USG guided or vacuumassisted core biopsy should be done. Due to restricted number of cases during the one year study, further work is needed with a longer duration of study and larger number of patients.

\section{REFERENCES}

1. Kambhoj A. Investigating a lump for suspected cancer: fine needle aspiration cytology. AIIMS 2007.

2. Smith MJ, Heffron CC, Rothwell JR, et al. Fine needle aspiration cytology in symptomatic breast lesions: still an important diagnostic modality? Breast J 2012; 18(2): 103-10.

3. Berner A, Davidson B, Sigstad E, et al. Fine-needle aspiration cytology vs. core biopsy in the diagnosis of breast lesions. Diagn Cytopathol 2003;29(6):344-8.

4. oyama T, Kibuchi Y, McKEE G. Core Needle biopsy as a diagnostic method for breast lesions, comparision with fine needle aspiration cytology. Breast Cancer 2004;11(4)339-42.

5. Ellis IO, Humphreys S, Michell M, et al. Guidelines for non-operative diagnostic procedures and reporting in breast cancer screening. NHS Cancer Screening Programme, Sheffield, UK 2001.

6. Garbar C, Cure H. FNA Cytology can play a role in neoadjuvant chemotherapy in operable breast cancer. ISRN Oncology Article ID- 935796, 2013;2013:1-5.

7. Bukhari MH, Arshad M, Jamal S, et al. Use of FNA in the evaluation of breast lumps. Pathology Research International Article ID- 689521, 2011;2011:1-10.

8. Panjavani SI, Parikh BJ, Parikh SB, et al. Utility of FNAC in the evaluation of breast lesions. Journal of Clinical and Diagnostic Research 2013;7(12)2777-9.

9. Vestito A, Mangieri FF, Gatta G, et al. Breast carcinoma in elderly women. Our experience. G Chir 2011;32(10):411-6.

10. Hussain MT. Comparison of fine needle aspiration cytology with excision biopsy of breast lump. J Coll Physicians Surg Pak 2005;15(4):211-4.

11. Brenner RJ, Parisky Y. Alternative breast-imaging approaches. Radiol Clin North Am 2007;45(5):907-23.

12. Capalbo E, Sajadidehkordi F, Colombi C, et al. Revaluation of breast cytology with pathologist on-site of lesions with suspicious sonographic features. Eur J Radiol 2013;82(9):1410-5.

13. Ibrahim AE, Bateman AC, Theaker JM, et al. The role and histological classification of needle core biopsy in comparison with fine needle aspiration cytology in the preoperative assessment of impalpable breast lesions. J Clin Pathol 2001;54(2):121-5.
14. Hatada $\mathrm{T}$, Ishii $\mathrm{H}$, Ichii $\mathrm{S}$, et al. Diagnostic value of ultrasound-guided fine-needle aspiration biopsy, coreneedle biopsy, and evaluation of combined use in the diagnosis of breast lesions. J Am Coll Surg 2000;190(3):299-303.

15. Barra Ade A, Gobbi H, de L Rezende CA, et al. A comparison of aspiration cytology and core needle biopsy according to tumour size of suspicious breast lesions. Diagn Cytopathol 2008;36(1):26-31.

16. Willems SM, van Deurzen CH, van Diest PJ. Diagnosis of breast lesions: fine-needle aspiration cytology or core needle biopsy? A review. J Clin Pathol 2012;65(4): 287-92.

17. Westenend PJ, Sever AR, Beekman-De Volder HJ, et al. A comparison of aspiration cytology and core needle biopsy in the evaluation of breast lesions. Cancer 2001;93(2):146-50.

18. Provenzano E, Pinder SE. Pre-operative diagnosis of breast cancer in screening: problems and pitfalls. Pathology 2009;41(1):3-17.

19. Parker SH, Burbank F, Jackman RJ, et al. Percutaneous large-core breast biopsy: a multi-institutional study. Radiology 1994;193(2):359-64.

20. He Q, Fan X, Yuan T, et al. Eleven years of experience reveals that fine-needle aspiration cytology is still a useful method for preoperative diagnosis of breast carcinoma. Breast 2007;16(3):303-6.

21. Hukkinen K, Kivisaari L, Heikkilä PS, et al. Unsuccessful preoperative biopsies, fine needle aspiration cytology or core needle biopsy, lead to increased costs in the diagnostic workup in breast cancer. Acta Oncol 2008;47(6):1037-45.

22. Manfrin E, Falsirollo F, Remo A, et al. Cancer size, histotype and cellular grade may limit the success of fine-needle aspiration cytology for screen-detected breast carcinoma. Cancer 2009;117(6):491-9.

23. Youk JH, Kim EK, Kim MJ, et al. Missed breast cancers at US-guided core needle biopsy: how to reduce them. Radiographics 2007;27(1):79-94.

24. Kalles V, Zografos GC, Provatopoulou X, et al. The current status of positron emission mammography in breast cancer diagnosis. Breast Cancer 2013;20(2):123-30.

25. Patel JJ, Gartell PC, Guyer APB, et al. Use of ultrasound localization to improve results of fine needle aspiration cytology of breast masses. J R Soc Med 1988;81(1):10-2.

26. Silverman JF, Elsheikh TM, Singh HK. The role of fine needle aspiration cytology of the breast in the core biopsy Era. Pathology case review 2007;12(1);44-8.

27. Homesh NA, Issa MA, El-Sofiani HA. The diagnostic accuracy of fine needle aspiration cytology versus core needle biopsy for palpable breast lump(s). Dept. of General Surgery, Sana'a University Sana'a, Yemen. Saudi Med J 2005;26(1):42-6.

28. Fervers B, Burgers JS, Haugh MC, et al. Predictors of high quality clinical practice guidelines: examples in oncology. International Journal for Quality in Health Care 2005;17(2):123-32.

29. Rosai J. Rosai and Ackermans surgical pathology. 9th edn. New York Mosby 2004.

30. Handa U, Kumar A, Kundu R, et al. Evaluation of grading and hormone receptor immunostaining on fine needle aspirates in carcinoma breast. J Cytol 2015;32(1):1-5. 